\title{
Polder maps: Improving OMIT maps for ligand building and validation
}

\author{
Dorothee Liebschnera, Pavel V. Afoninea, Nigel W. Moriartya, Billy K. Poonª, Oleg V. \\ Soboleva, and Paul D. Adams ${ }^{\text {ab }}$ \\ aMolecular Biophysics \& Integrated Bioimaging Division, Lawrence Berkeley \\ National Laboratory (LBNL), Berkeley, CA, 94720, USA \\ bDepartment of Bioengineering, University of California Berkeley, Berkeley, \\ California, 94720, USA
}

In macromolecular crystallography, electron density maps are used to build and validate crystallographic models. In particular, OMIT maps ${ }^{1}$ are a common tool to verify the presence of ligands in a crystal structure. The simplest way to compute an OMIT map is to exclude the ligand from the model, update the structure factors and compute a residual map. It is then expected that if the ligand is present in the crystal structure, the electron density for the omitted atoms will appear as positive features in this map. However, this is complicated by the flat bulk-solvent model ${ }^{2}$, which postulates constant electron density in the areas of the unit cell that are not occupied by the atomic model. Thus, if atoms are removed from the model, the region where they were modelled will be filled with bulk-solvent, and if the density arising from the omitted atoms is weak, then the bulk-solvent model may obscure it further.

A possible solution to this problem is to prevent bulk-solvent from entering the OMIT regions, which may improve the interpretative power of residual maps. This approach is called polder (OMIT) map $^{3}$ and the tool is implemented and available in the software suite Phenix ${ }^{4}$. Polder OMIT maps can be particularly useful for displaying weak densities of ligands. Several examples are presented where polder OMIT maps show clearer features than conventional OMIT maps.

1. Bhat, T. N. \& Cohen, G. H. Omitmap: an Electron Density Map Suitable for the Examination of Errors in a Macromolecular Model. J. Appl. Crystallogr. 17, 244-248 (1984).

2. Jiang, J. \& Brünger, A. Protein hydration observed by X-ray diffraction. J. Mol. Biol 243, 100 115 (1994).

3. Liebschner, D. et al. Polder maps: Improving OMIT maps by excluding bulk-solvent. Acta Crystallogr. Sect. D Biol. Crystallogr. 73, 148-157 (2017).

4. Adams, P. D. et al. PHENIX: A comprehensive Python-based system for macromolecular structure solution. Acta Crystallogr. Sect. D Biol. Crystallogr. 66, 213-221 (2010). 